\title{
Pro-survival responses to the dual inhibition of anti-apoptotic Bcl-2 family proteins and mTOR-mediated signaling in hypoxic colorectal carcinoma cells
}

Karianne Risberg ${ }^{1,2}$, Kathrine Røe Redalen ${ }^{1}$, Linda Sønstevold ${ }^{1}$, Tonje Bjørnetrø $\varnothing^{1,3}$, Janne Sølvernes ${ }^{1}$ and Anne Hansen Ree ${ }^{1,3^{*}}$

\begin{abstract}
Background: The use of targeted agents to impel dual inhibition of anti-apoptotic mechanisms and mTORmediated pro-survival signaling in colorectal carcinoma (CRC) cell lines with KRAS or BRAF mutation has been shown to induce apoptosis, a timely result given CRC entities harboring such mutations are in need of new therapies. Since CRC comprises heterogeneous tumors with predominant hypoxic components, we investigated effects of an inhibitor of anti-apoptotic Bcl-2 family proteins (ABT-737) in combination with an mTOR inhibitor (AZD8055)—collectively referred to as combo-Rx, in hypoxic CRC cell lines.

Methods: Cell viability measures, expression of proteins implicated in apoptosis and MAPK/PI3K-AKT/mTOR pathway signaling, and profiling of composite kinase activities were undertaken in a panel of 14 cell lines.

Results: In hypoxic conditions, combo-Rx suppressed viability of 13 of the cell lines, albeit ABT-737 did not significantly potentiate the inhibitory effect of single-agent AZD8055 in six of the models. Hypoxic KRAS/PIK3CA-mutant HCT-116 and HCT-15 cell lines (both with low endogenous expression of the anti-apoptotic MCl-1 protein and showing augmented inhibition of viability following the addition of ABT-737 to AZD8055) responded to combo-Rx by induction of apoptosis but with the simultaneous strong Mcl-1 up-regulation and activation of MAPK/PI3K-conducted signaling. In contrast, in hypoxic KRAS-mutant LoVo (devoid of PIK3CA mutation), BRAF/PIK3CA-mutant RKO, and wild-type Colo320DM cell lines (all with high endogenous Mcl-1 expression and being resistant to the additional effect of ABT-737 to AZD8055), combo-Rx did not elicit apoptotic or pro-survival responses.

Conclusions: The concurrent inhibition of anti-apoptotic proteins and mTOR-mediated signaling in hypoxic KRAS/ PIK3CA-mutant CRC cell lines resulted in pro-survival responses in parallel with the intended anti-proliferative effects, a finding that should be of note if considering combinatory targeting of multiple pathways in this CRC entity.
\end{abstract}

Keywords: ABT-737, Apoptosis, AZD8055, Colorectal cancer, Hypoxia, Kinase activity, KRAS, Mcl-1, PIK3CA

\footnotetext{
* Correspondence: a.h.ree@medisin.uio.no

${ }^{1}$ Department of Oncology, Akershus University Hospital, 1478 Lørenskog,

Norway

${ }^{3}$ Institute of Clinical Medicine, University of Oslo, 0318 Oslo, Norway

Full list of author information is available at the end of the article
} 


\section{Background}

Colorectal carcinoma (CRC), like most solid malignancies, comprises heterogeneous tumors with predominant hypoxic components. The adaptive tissue responses to hypoxic stress involve increased resistance to apoptosis (programmed cell death) as well as altered DNA damage repair and mutation rates, and thereby genomic instability [1-3], ultimately leading to compromised efficacy of DNA-damaging therapies (chemotherapy and radiation). Moreover, mutations in genes such as KRAS, BRAF, and $P I K 3 C A$ commonly result in constitutive activation of cellular signaling mediated by mitogen-activated protein kinases (MAPK) and phosphatidylinositol 3-kinase-protein kinase B (PI3K-AKT) [4, 5]. These pathways converge at the mechanistic target of rapamycin (mTOR), which regulates cell growth and survival [6] and makes the mTOR complex an attractive target for CRC therapy. Consequently, a number of mTOR inhibitors have entered clinical trials.

There is however evidence of crosstalk between the mTOR-conducted signaling and other signaling pathways which will allow tumor cells to escape mTOR-inhibitory therapy $[7,8]$. Targeting of multiple pathways has therefore been considered. Recent findings showed that the combination of the mTOR inhibitor AZD8055 with ABT-263, an inducer of apoptosis, promoted cell death in CRC cell lines with $K R A S$ or $B R A F$ mutation [9], a timely result given CRC entities harboring these mutations are refractory to current targeted therapies. ABT-263 and its structurally related compound ABT-737 are potent inhibitors of the anti-apoptotic proteins Bcl-2, Bcl-xL, and Bcl-w, but not of Mcl-1, and induce apoptosis in cancer cells [10, 11]. Overexpression of Mcl-1 is associated with resistance to ABT737, and inhibition of Mcl-1 has proven to sensitize cancer cells to ABT-737 [12-14]. Interestingly, hypoxia has been shown to promote ABT-737-mediated apoptotic cell death in small-cell lung carcinoma, CRC, and hematologic cell lines via down-regulation of Mcl-1 [15-17].

Since no information is available regarding the concurrent inhibition of anti-apoptotic proteins and mTORmediated pro-survival signaling under CRC tumor hypoxia, we investigated response to treatment with ABT-737 and AZD8055, in this report referred to as combo- $\mathrm{Rx}$, in a panel of hypoxic CRC cell lines harboring various typical mutations.

\section{Methods}

\section{Cell lines, culture conditions, and reagents}

Fourteen human CRC cell lines (kindly provided by Prof. Kjersti Flatmark, Oslo University Hospital, Oslo, Norway or purchased from the American Type Culture Collection, Manassas, VA, USA) were first determined for mutations in KRAS, BRAF, and PIK3CA by Ion Torrent PGM ${ }^{\text {тм }}$ sequencing, and mutation profiles were in agreement to already published data [18-20]. All cell lines except Caco2 were kept in RPMI 1640 medium (Sigma-Aldrich, St. Louis, MO, USA) supplemented with $10 \%$ fetal bovine serum (Gibco by Life Technologies, Grand Island, NY, USA) and $2 \mathrm{mM}$ L-glutamine (GE Healthcare, PAA Laboratories, Pashing, Austria). The Caco-2 cells were kept in DMEM medium (Sigma-Aldrich) containing $15 \%$ serum. The cell lines were routinely tested and found free of mycoplasma infection. For all assays, cells were seeded and left to adhere overnight to reach exponential growth at start of experiments. Cells were incubated under normoxic $\left(21 \% \mathrm{O}_{2}\right)$ or hypoxic $\left(0.2 \% \mathrm{O}_{2}\right)$ conditions, the latter obtained using the hypoxic chamber Invivo2 300 (Ruskinn Technologies, Leeds, UK). The mTOR inhibitor AZD8055, the PI3K/mTOR inhibitor BEZ235, the Bcl-2 family protein inhibitor ABT-737, and the pan-caspase inhibitor Z-VAD (all by Selleckchem.com, SMS-gruppen, Rungsted, Denmark) were dissolved in dimethyl sulfoxide (Sigma-Aldrich). Control cells received the vehicle.

\section{Cell viability assay}

Depending on the cell line, 12,000-20,000 cells were seeded per well in 96-well Costar plates (Corning Incorporated, Corning, NY, USA). Cells were given ABT-737 or AZD8055, separately or combined, in increasing concentrations $(0.10-10 \mu \mathrm{M}$; combo-Rx designates $10 \mu \mathrm{M}$ of both compounds), the combination of ABT-737 and BEZ235 (10 $\mu \mathrm{M}$ of both compounds), or vehicle. When expedient, the cells were pre-treated for 45 min with Z-VAD (20 or $50 \mu \mathrm{M})$. Cell viability was determined after 24 or $72 \mathrm{~h}$ by adding CellTiter $96^{\circ} \mathrm{AQ}$ ueous One Solution Reagent according to the manufacturer's instructions (the MTS assay; Promega, Madison, WI, USA). Absorbance was measured using Varioscan (Thermo Electron, Waltham, MA, USA). Values were corrected for background absorbance, and values for treated cells are reported as percentage cell viability to corresponding control cell values. Presented results are from between three and seven independent experiments, each plated at least in triplicate.

\section{Western blot analysis}

Cells were seeded in Nuncleon T25 flasks (Thermo Fisher Scientific, Roskilde, Denmark) and were treated as indicated, and protein lysates from both floating and adherent cells were harvested as previously described [21]. Equal amounts of protein $(20 \mu \mathrm{g})$ were separated by NuPAGEBisTris (Novex by Life Technologies, Carlsbad, CA, USA), transferred by electrophoresis to Immobilon ${ }^{\circledR}$ membrane (Millipore Corporation, Billerica, MA, USA), and probed with antibodies against hypoxia-inducible factor type $1 \alpha$ (HIF-1 $\alpha$; BD Transduction Laboratories, Franklin Lakes, NJ, USA) and carbonic anhydrase IX (CAIX; kindly provided by Prof. Silvia Pastorekova, Slovak Academy of Sciences, Bratislava, Slovak Republic), and against Mcl-1, 
Bcl-2, Bcl-xL, caspase-3, mitogen-activated protein kinase3/1 (ERK1/2), p-ERK1/2(Thr202/Tyr204), AKT, pAKT(Ser473), ribosomal protein S6 kinase beta-2 (S6), and p-S6(Ser235/236) (Cell Signaling Technology, La Jolla, CA, USA). Anti- $\alpha$-tubulin (Calbiochem/EMD Chemicals Inc., San Diego, CA, USA) and Amido Black (Sigma-Aldrich) total protein staining were used as loading controls. Secondary antibodies were from Dako Denmark AS (Glostrup, Denmark). Peroxidase activity was visualized using SuperSignal West Dura Extended Substrate (Thermo Scientific, Rockford, IL, USA). Sufficient amount of lysate from each sample was prepared to run three gels. The parallel blotting membranes were considered identical, and different proteins were visualized on different membranes for practicality. All Western blot experiments were performed as three biological replicates.

\section{RNA interference}

Mcl-1 expression was inhibited using short hairpin (sh)RNA (clone ID NM_021960.3-953s1c1; Sigma-Aldrich), and control cells were generated using non-target sequence (product number shc002v; Sigma-Aldrich). The manufacturer's instructions were followed apart from extending the lentiviral incubation period to $48 \mathrm{~h}$.

\section{Microscopy}

Cells were seeded in Nuncleon T25 flasks and treated as indicated for up to $72 \mathrm{~h}$. When expedient, the cells were pre-treated for $45 \mathrm{~min}$ with Z-VAD. Phasecontrast images were processed at the start of experiment and further after 24, 48, and $72 \mathrm{~h}$ by Olympus IX81 (Olympus Europa Holding GmbH, Hamburg, Germany).

\section{Kinase activity profiling}

The Tyrosine Kinase PamChip ${ }^{\circ}$ Array technology (PamGene International B.V., 's-Hertogenbosch, The Netherlands) enables profiling of composite tissue kinase activities [4]. The array contains peptides that are kinase substrates and consisting of 13 or 14 amino acids with tyrosine residues for phosphorylation. Protein lysates used for Western blot analysis were also incubated on the arrays for kinase activity profiling. Substrate phosphorylation intensities were measured using the Evolve software (PamGene International B.V.). Applying BioNavigator software (PamGene International B.V.), endpoint signal intensities generated from bound fluorescent antiphosphotyrosine antibody were converted to numerical values. The primary array data are available in the ArrayExpress data repository (http://www.ebi.ac.uk/ arrayexpress/experiments/E-MTAB-3870/) by accession number E-MTAB-3870. Background signals were subtracted, and negative signal intensities were managed by subtracting the $1 \%$ quantile of all data and setting the remaining signal intensities less than 1 to the value of 1 . Data were $\log _{2}$-transformed before mean signal intensity of three replicates that were analyzed for each experimental condition was calculated for each peptide substrate. The resulting data from each type of treatment was compared to the relevant control (vehicle-treated cells) for assessment of increase or decrease in substrate phosphorylation level. Substrates associated with PI3K-AKT and/or MAPK pathways were retrieved from PathCards (http:// pathcards.genecards.org/), applying the super-pathway definitions 'PI3K-AKT signaling pathway' and 'MAPK signaling pathway'.

\section{Statistical analysis}

Differences between groups were analyzed using two-tailed Student's $t$-test. $p$-values less than 0.05 were considered statistically significant. In the assessment of combination effects on cell viability, we chose to evade calculations based on median-effect equation for multiple drug interactions because the ABT-737 single-agent effects did not appear with typical dose-response curves.

\section{Results \\ Inhibition of anti-apoptotic proteins or mTOR-mediated signaling-cell viability}

First, individual effects of the $\mathrm{Bcl}-2$ family protein inhibitor ABT-737 and the mTOR inhibitor AZD8055 on cell viability under hypoxia and normoxia at $24 \mathrm{~h}$ were examined (Additional file 1: Fig. S1a). Incubation of HCT-116, RKO, HT-29, and Colo320DM cell lines with increasing concentrations of ABT-737 (0.10-10 $\mu \mathrm{M})$ had modest effects. In contrast, differential sensitivity was observed with AZD8055 (0.10-10 $\mu \mathrm{M})$, with HT29 cells being refractory and HCT-116, RKO, and Colo320DM cells displaying suppressed cell viability in the order of 20-60\% depending on the oxygenation status. The intended cellular response to hypoxia was confirmed by the time-dependent induction of HIF- $1 \alpha$ and its target CAIX in HCT-116 cells (Additional file 1: Fig. S1b).

Since Faber and co-workers found that the combination of ABT-263, a structurally related compound to ABT-737, with AZD8055 at concentrations of 50-500 nM for $72 \mathrm{~h}$ caused apoptosis in CRC cell lines with $K R A S$ or $B R A F$ mutation [9], we next investigated inhibitory effects on cell viability when combining AZD8055 at $0.10 \mu \mathrm{M}$ with ABT-737 for 72 h. For seven tested cell lines, ABT-737 potentiated AZD8055 under hypoxia in only one of five mutant ones (of which three and two had KRAS and $B R A F$ mutation, respectively; one of each had also PIK3CA mutation) but in both of the wild-type models (Additional file 2: Tables S1a and S1b). 


\section{Combo-Rx and hypoxic cell viability}

Based on the two initial sets of results, the highest tested concentration $(10 \mu \mathrm{M})$ of both compounds was chosen for further experiments. First, we investigated viability in 14 CRC cell lines under normoxic conditions (Additional file 2: Table S2). The addition of ABT-737 significantly potentiated inhibitory effects of AZD8055 in four of six KRAS-mutant and one of three wild-type cell lines but in none of five $B R A F$-mutant models. The findings in the two first-mentioned groups but not in BRAF-mutant cell lines were generally in agreement with previously reported data [9].

Specifically, we were interested in elucidating inhibitory effects of combo-Rx under hypoxic conditions in the 14 cell lines (Fig. 1). Again, single-agent ABT-737 caused limited decrease in cell viability (median decline of $6.2 \%$ (range, -2.0 to $19 \%$ ) across cell lines), which was significant in five cell lines only. Incubation with AZD8055 (by itself resulting in a median decline of $25 \%$ (range, -7.0 to $45 \%$ ) across the cell lines) or combo-Rx significantly suppressed cell viability in 11 and 13 of the 14 cell lines, respectively. However, as shown in Table 1, in six of the hypoxic cell lines (one of six KRAS-mutant, three of five BRAF-mutant, and two of three wild-type ones), ABT-737 did not significantly potentiate the inhibitory effect of single-agent AZD8055. Three of the 14 cell lines, two sensitive and one resistant to the additional effect of ABT-737, had PIK3CA mutation. Similarly, treatment with the PI3K/mTOR inhibitor BEZ235, which has demonstrated anti-proliferative effects in various models [22, 23], caused a median decline of $22 \%$ (range,
3.5 to $47 \%$ ) in cell viability across hypoxic cell lines, which in five of 12 cell lines was not significantly potentiated by ABT-737 (Additional file 2: Tables S3a and S3b).

\section{Combo-Rx-Mcl-1 and apoptotic response}

Further focusing on the finding that ABT-737 was unable to significantly potentiate the effect of AZD8055 on cell viability in approximately half of hypoxic cell lines investigated and moreover, since previous studies have shown that suppression of the anti-apoptotic Mcl-1 protein sensitizes cells to ABT-737 [12-14], Mcl-1 was knocked down in the KRAS/PIK3CA-mutant HCT-116 cell line (with low endogenous Mcl-1 expression and being sensitive to the additional effect of ABT-737) and the BRAF/PIK3CA-mutant RKO and wild-type Colo320DM cell lines (both with high endogenous Mcl-1 expression and being resistant to the additional effect of ABT-737) (Fig. 2a). Specific knockdown of Mcl-1, though not complete, and not of other anti-apoptotic proteins was confirmed (Fig. 2b). As shown in Fig. 2c, Mcl-1 knockdown significantly sensitized for inhibitory cell viability effects by ABT-737 and combo-Rx in all three hypoxic cell lines. Next, when the Mcl-1-repressed cell lines were pre-treated with the pan-caspase inhibitor Z-VAD $(20 \mu \mathrm{M})$ in order to examine to which extent apoptosis might account for responses, the inhibitory cell viability effect was completely abolished in hypoxic shMcl-1 HCT-116 cells given ABT-737 or combo-Rx and partly counteracted by combo-Rx in hypoxic shMcl-1 RKO cells (Fig. 2d). On increasing the Z-VAD concentration to $50 \mu \mathrm{M}$, no further regulatory effects were seen in the

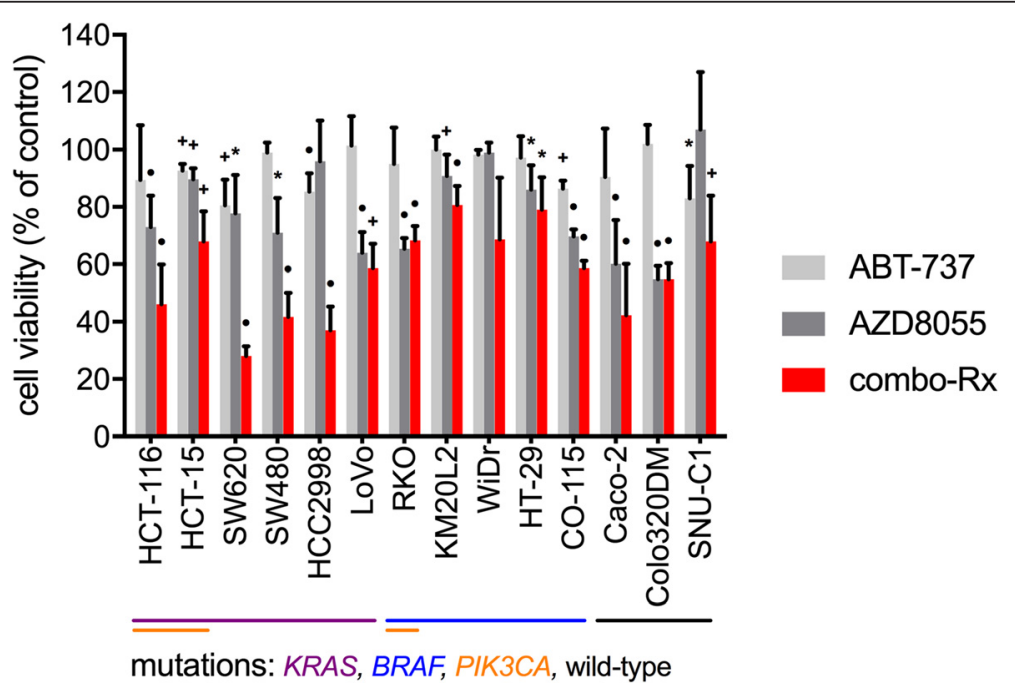

Fig. 1 Cell viability in hypoxic colorectal carcinoma (CRC) cell lines. Fourteen CRC cell lines were treated for $24 \mathrm{~h}$ with ABT-737 (inhibitor of antiapoptotic Bcl-2 family proteins; $10 \mu \mathrm{M}$ ), AZD8055 (mTOR inhibitor; $10 \mu \mathrm{M}$ ), or combo-Rx (10 $\mu \mathrm{M}$ of both compounds in combination) under hypoxic conditions. Cell viability (measured by the MTS assay) value for each condition relative to the corresponding control cell value is shown as mean \pm SD. Statistically significant changes are indicated (asterisk, $p<0.05$; cross, $p<0.01$; circle, $p<0.001$ ) 
Table 1 Cell viability of hypoxic human colorectal carcinoma cell lines

\begin{tabular}{llllll}
\hline Cell line & Mutations & ABT-737 & AZD8055 & combo-Rx & $p$-value \\
\hline HCT-116 & KRAS, PIK3CA & $89.4 \pm 19.1$ & $73.0 \pm 10.9$ & $46.1 \pm 13.7$ & 0.0016 \\
HCT-15 & KRAS, PIK3CA & $92.7 \pm 2.31$ & $89.7 \pm 3.79$ & $68.0 \pm 10.4$ & 0.028 \\
SW620 & KRAS & $80.6 \pm 8.91$ & $77.8 \pm 13.4$ & $28.0 \pm 3.46$ & $<0.001$ \\
SW480 & KRAS & $99.0 \pm 3.46$ & $71.0 \pm 12.1$ & $41.7 \pm 8.39$ & 0.026 \\
HCC2998 & KRAS & $85.3 \pm 6.41$ & $96.0 \pm 14.2$ & $37.0 \pm 8.37$ & $<0.001$ \\
LoVo & KRAS & $101 \pm 10.3$ & $64.0 \pm 7.21$ & $58.7 \pm 8.50$ & 0.45 \\
RKO & BRAF, PIK3CA & $95.0 \pm 12.8$ & $65.3 \pm 3.79$ & $68.3 \pm 5.03$ & 0.46 \\
KM20L2 & BRAF & $100 \pm 4.62$ & $90.9 \pm 7.38$ & $80.7 \pm 6.63$ & 0.019 \\
WiDr & BRAF & $98.3 \pm 1.71$ & $99.0 \pm 3.46$ & $68.7 \pm 21.6$ & 0.074 \\
HT-29 & BRAF & $97.3 \pm 7.48$ & $86.0 \pm 8.54$ & $79.0 \pm 11.4$ & 0.44 \\
CO-115 & BRAF & $86.3 \pm 2.89$ & $69.7 \pm 2.52$ & $58.7 \pm 2.52$ & 0.0059 \\
Caco-2 & wild-type & $90.4 \pm 17.0$ & $60.1 \pm 15.3$ & $42.3 \pm 17.9$ & 0.068 \\
Colo320DM & wild-type & $102 \pm 6.73$ & $54.9 \pm 4.56$ & $54.9 \pm 5.46$ & 1.0 \\
SNU-C1 & wild-type & $83.0 \pm 11.4$ & $107 \pm 20.0$ & $68.0 \pm 15.9$ & 0.023
\end{tabular}

From between three and seven independent experiments, each at least with triplicate setups, mean $\pm S D$ cell viability values were calculated in percentage of values from the corresponding controls (vehicle-treated cells). Treatments (24-h incubations): ABT-737 (inhibitor of anti-apoptotic Bcl-2 family proteins; $10 \mu \mathrm{M}$ ), AZD8055 (mTOR inhibitor; $10 \mu \mathrm{M}$ ), combo-Rx (10 $\mu \mathrm{M}$ of both compounds in combination). Difference in values from cells given AZD8055 or combo-Rx was compared by two-tailed Student's $t$-test. Mutation status is indicated for each cell line

Mcl-1-repressed RKO and Colo320DM cell lines (data not shown). These results do not exclude the possibility that the low levels of remaining Mcl-1 protein were able to protect these cell lines to inhibitory effects of ABT737, as complete knockdown of Mcl-1 was not obtained (Fig. 2b). Nevertheless, the data collectively indicated two different modes of hypoxic cell viability response, one clearly involving apoptosis (in HCT-116 cells) and another not.

To explore the notion of non-apoptotic mechanisms of combo-Rx effects in RKO and Colo320DM cell lines, the three hypoxic cell lines were treated for $72 \mathrm{~h}$ and inspected at 24-h intervals (Additional file 3: Fig. S2). In HCT-116 cells, a mixture of pycnotic cells indicating apoptosis [24] and still surface-attached cells was observed at $24 \mathrm{~h}$. This particular finding of a dual phenotype remained for the entire incubation period of $72 \mathrm{~h}$ (data not shown). In the Colo320DM and RKO cell lines, combo-Rx caused decrease in cell confluence, indicating growth-inhibitory effects, but essentially few pycnotic cells after 48 and $72 \mathrm{~h}$, respectively. The Colo320DM cells did not tolerate the combo-Rx drug concentrations in hypoxia for $72 \mathrm{~h}$. In neither of the two cell lines, the growth inhibition following combo-Rx was abolished by Z-VAD (data not shown), again arguing against the involvement of apoptosis.
Combo-Rx-activation of hypoxic pro-survival signaling Following the observations in hypoxic HCT-116 cells that combo-Rx was effective in inhibiting viability but caused both surface-attached and pycnotic cells, underlying mechanisms for the regulatory effects were investigated. The HCT-116, RKO, and Colo320DM cell lines from the preceding sets of experiments were complemented with HCT-15 cells (KRAS/PIK3CA-mutant and sensitive to the additional effect of ABT-737 to ABT8055) and LoVo cells (with high endogenous Mcl-1 expression (Fig. 2a) and the only KRAS-mutant model that was resistant to combo- $R x$ for viability). The five cell lines were assessed for induction of apoptosis, as indicated by cleavage of caspase- 3 , and for pro-survival signaling by phosphorylation of ERK1/2 (p-ERK1/2) of the MAPK pathway, of AKT (p-AKT(Ser473)) of the PI3K-AKT pathway, and of S6 (p-S6) down-stream of mTOR, and by expression of the anti-apoptotic protein Mcl-1 (Fig. 3).

Of initial note, $24 \mathrm{~h}$ of hypoxic incubation in itself (i.e., of control cells) inhibited Mcl-1 expression in the majority of the cell lines. Although levels of caspase-3 were also lower under hypoxic compared to normoxic conditions in both cell lines that were sensitive to the additional effect of ABT-737 to ABT8055 (HCT-116 and HCT-15), cleaved caspase-3 was observed after $24 \mathrm{~h}$ of hypoxic combo-Rx. As expected, this apoptotic feature could not be detected in the three resistant cell lines (LoVo, RKO, and Colo320DM). Next, under normoxia, ABT-737 treatment caused Mcl-1 up-regulation and $\mathrm{p}-\mathrm{S} 6$ induction as a consequence, and these responses were counteracted by the addition of AZD8055 (i.e., under combo-Rx compared to ABT-737 treatment alone). These observations were in agreement with previously reported data [9]. Under hypoxia also, Mcl-1 up-regulation by ABT-737 alone was generally seen in all cell lines with mutations (HCT-116, HCT-15, LoVo, and RKO); however, a striking response followed the addition of AZD8055 to ABT-737. The two models that showed potentiation with combo-Rx (HCT-116 and HCT-15; both also with PIK3CA mutation) demonstrated a further strong induction of Mcl-1 and $\mathrm{p}-\mathrm{S} 6$ and also of p-ERK1/2. In contrast, hypoxic combo-Rx did not cause any uniform pattern of regulation of these selected pro-survival factors in the resistant cell lines. Specifically, in the KRAS-mutant LoVo cell line (devoid of PIK3CA mutation), the addition of AZD8055 counteracted the effects of ABT-737 on Mcl-1 and pS6, exactly like under normoxia. The hypoxic BRAF/ PIK3CA-mutant RKO cells showed Mcl-1 and p-S6 responses similar to but not as strong as those of the HCT-116 and HCT-15 models. And in the wild-type Colo320 cell line, hypoxic combo-Rx did not cause Mcl-1 alteration nor was p-S6 detected. In summary, 


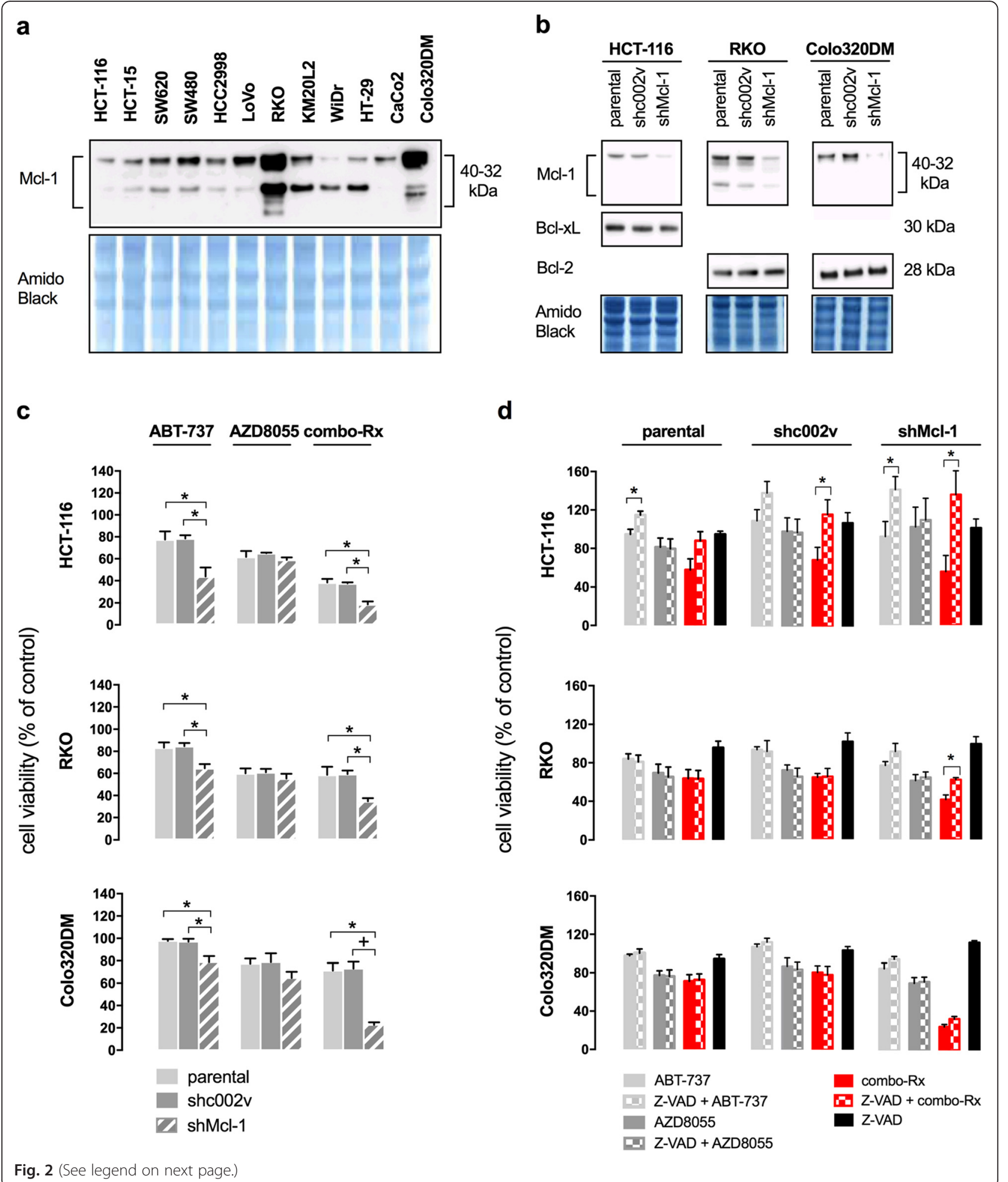


(See figure on previous page.)

Fig. 2 The anti-apoptotic Mcl-1 protein and hypoxic cell viability. a Expression of Mcl-1 in a panel of human colorectal carcinoma cell lines, as illustrated by Western blot analysis. The experiment was performed twice. $\mathbf{b}$ Three parental cell lines were treated with short hairpin (sh)RNA of Mcl-1 and a non-target control sequence (shc002v), and expression of anti-apoptotic proteins was examined by Western blot analysis. The experiment was performed at least three times for each of the cell line groups. c The parental and the shc002v and shMcl-1 versions of the three cell lines were treated for $24 \mathrm{~h}$ with ABT-737 (inhibitor of anti-apoptotic Bcl-2 family proteins; $10 \mu \mathrm{M}$ ), AZD8055 (mTOR inhibitor; $10 \mu \mathrm{M}$ ), or combo-Rx (10 $\mu \mathrm{M}$ of both compounds in combination) under hypoxic conditions. Cell viability (measured by the MTS assay) values for treated cells relative to the corresponding controls (mean \pm SD) are shown. Statistically significant differences between cell line versions are indicated (asterisk, $p<0.05 ;$ cross, $p<0.01$ ). $\mathbf{d}$ The same cell line entities were treated for $24 \mathrm{~h}$ under hypoxic conditions with ABT-737, AZD8055, or combo-Rx alone or following pre-treatment for 45 min with the pan-caspase inhibitor Z-VAD $(20 \mu \mathrm{M})$, as indicated. Cell viability values relative to the corresponding control values are shown as mean \pm SD. Statistically significant differences between treatment groups are indicated (asterisk, $p<0.05$; cross, $p<0.01$ )

the results highlighted the possibility of dual apoptotic and pro-survival responses in KRAS/PIK3CA-mutant CRC cells responding under hypoxic conditions with augmented inhibition of viability to the addition of ABT-737 to AZD8055.

\section{Combo-Rx - activation of hypoxic kinase signaling}

Following the intriguing results that phosphorylation of a discrete number of MAPK/mTOR-signaling mediators was induced by combo-Rx in hypoxic KRAS/PIK3CA-mutant cell lines, we applied the Tyrosine Kinase PamChip Array to assess HCT-116 kinase activities more broadly. The KRAS-mutant HCC2998 cell line, which also responded to hypoxic combo-Rx with augmented inhibition of viability but did not harbor PIK3CA mutation (Table 1), and the non-responding wild-type Colo320DM cell line, in which
p-ERK1/2 and p-S6 had not been detected (Fig. 3), were analyzed for comparison.

As depicted in Fig. 4 and detailed in Additional file 4: Table S4, ABT-737 inhibited the kinase activity in both hypoxic and normoxic HCT-116 cells. Intriguingly, lysates from hypoxic HCT-116 cells given AZD8055 or combo$\mathrm{Rx}$ generated strong phosphorylation of the majority of the array peptides and not explicitly substrates associated with the MAPK or PI3K-AKT signaling pathways. In particular, peptides representing proteins involved in the angiogenic response to hypoxia (such as PDGFRB, EPOR, ANXA2, CTTN1, MET, PXN, and PECAM1) and MAPK/ PI3K-conducted signaling in particular (RASA1, RAF1, PIK3R1, and PDPK1) were highly phosphorylated ( $\log _{2}$ fold-change >2.0; Additional file 4: Table S4). In normoxic HCT-116 cells, treatment with AZD8055 generally

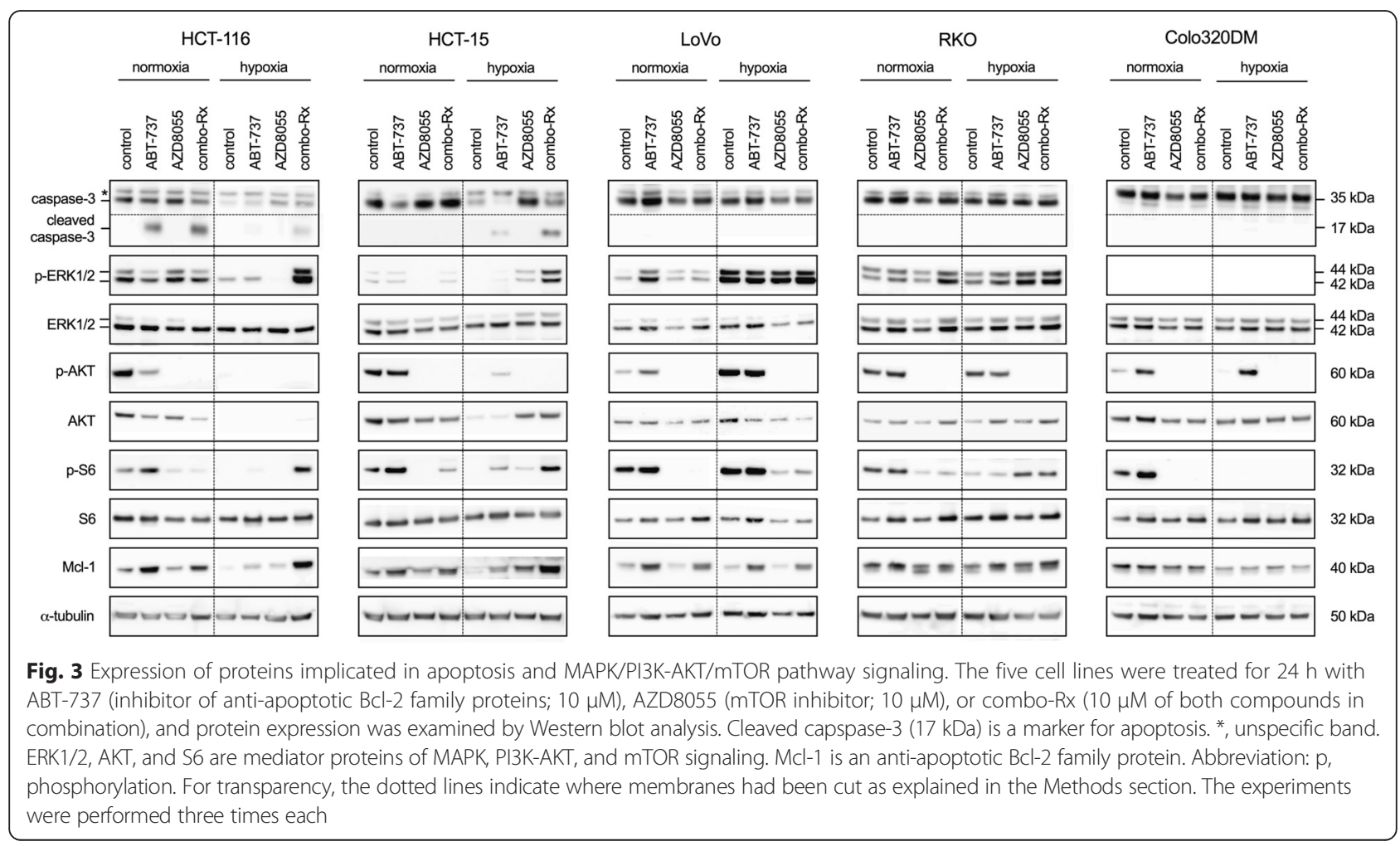




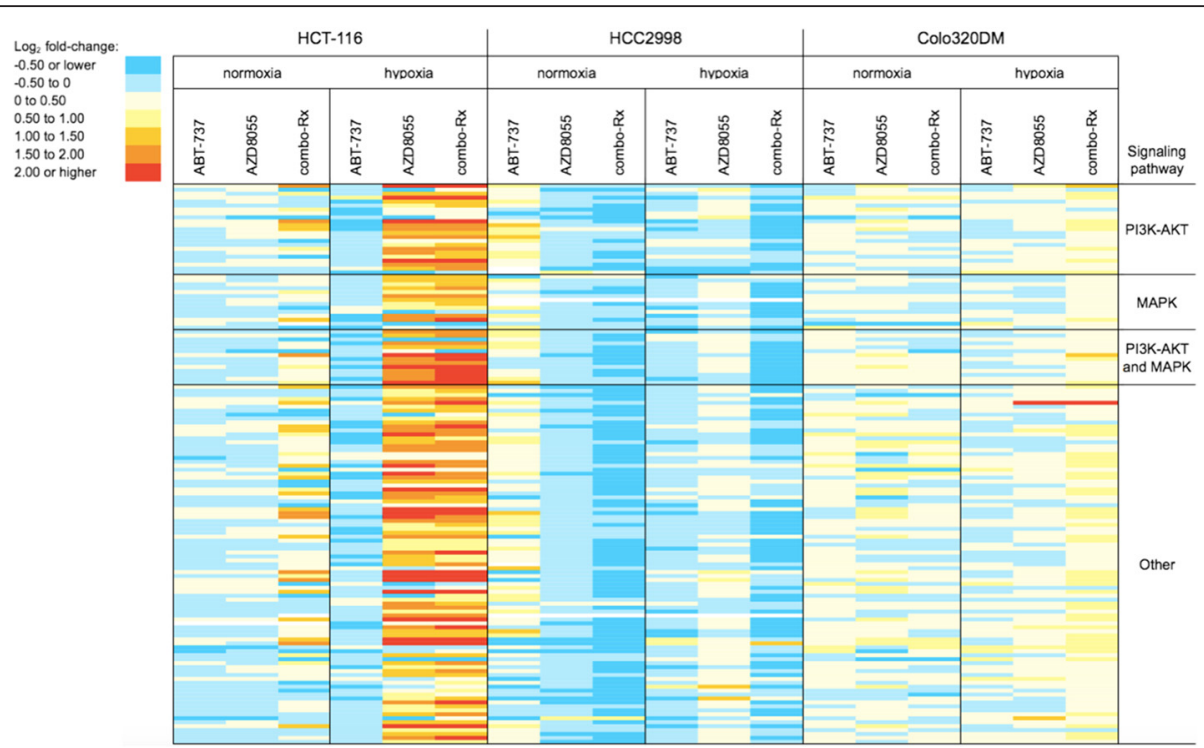

Fig. 4 Ex vivo kinase substrate phosphorylation. The color map visualizes normalized $\log _{2}$-transformed signal intensities from kinase substrate arrays incubated with lysates from the three cell lines treated for $24 \mathrm{~h}$ with ABT-737 (inhibitor of anti-apoptotic Bcl-2 family proteins; $10 \mu \mathrm{M}$ ), AZD8055 (mTOR inhibitor; $10 \mu \mathrm{M}$ ), or combo-Rx (10 $\mu \mathrm{M}$ of both compounds in combination). As quantified and categorized by the color codes, red corresponds to higher and blue to lower substrate phosphorylation levels relative to levels from the corresponding control cells (vehicle-treated). Substrates associated with PI3K-AKT and/or MAPK signaling pathways were retrieved from PathCards (http://pathcards.genecards.org/), applying the super-pathway definitions 'PI3K-AKT signaling pathway' and 'MAPK signaling pathway'. The identity of each peptide substrate, vertically in order from top to bottom, is given in Additional file 4: Table S4

suppressed kinase activity, while combo-Rx caused increased phosphorylation (but by $\log _{2}$ enhancement $<2.0$ ) of a limited number of substrates. In contrast, kinase activity of HCC2998 cells, whether hypoxic or normoxic, was predominantly repressed by all treatment conditions, while Colo320DM kinase activity was basically unchanged.

\section{Discussion}

A number of recent findings $[4,9,16,17]$ led us to investigate the effects of ABT-737, an inhibitor of anti-apoptotic Bcl-2 family proteins, in combination with the mTOR inhibitor AZD8055 in a panel of 14 hypoxic CRC cell lines. Combo-Rx (i.e., the combination treatment) suppressed viability of 13 of the cell lines, albeit ABT-737 did not significantly potentiate the inhibitory effect of singleagent AZD8055 in six of the models. On further mechanistic investigations, the hypoxic KRAS/PIK3CA-mutant HCT-116 and HCT-15 cell lines (both with low endogenous expression of the anti-apoptotic Mcl-1 protein and showing augmented inhibition of viability following the addition of ABT-737 to AZD8055) responded to combo$\mathrm{Rx}$ by induction of apoptosis (as assessed by various experimental approached in the HCT-116 cells) and with the simultaneous strong Mcl-1 up-regulation and activation of MAPK/PI3K-conducted signaling. A ubiquitous activation of hypoxic kinase signaling by combo-Rx was also confirmed in the HCT-116 cells. In contrast, in hypoxic
KRAS-mutant LoVo, BRAF/PIK3CA-mutant RKO, and wild-type Colo320DM cell lines (all with high endogenous Mcl-1 expression and being resistant to the additional effect of ABT-737 to AZD8055), combo-Rx did not elicit apoptotic or pro-survival responses. Collectively, this data revealed complex responses to the concurrent inhibition of anti-apoptotic proteins and mTOR-mediated signaling in hypoxic CRC cell lines, where pro-survival responses were elicited in parallel with the intended anti-proliferative effects in KRAS/PIK3CA-mutant entities in particular, a finding that should be of note if considering the combinatory targeting of multiple pathways in CRC treatment.

As recently shown [9], when combined with ABT-263 (structurally related to ABT-737), AZD8055 via the specific suppression of Mcl-1 sensitized CRC cell lines with KRAS or $B R A F$ mutation to undergo apoptosis, a timely result as such CRC entities are refractory to current targeted therapies. In vitro studies have shown promising treatment effects of both types of agents, but concerns have been raised with regard to lacking therapeutic efficacy of mTOR inhibitors in solid tumors $[11,25]$. Both AZD8055 and the antiapoptotic inhibitors ABT-263 and ABT-737 have reached early-phase clinical trials. However, only a few reports exist on the use of ABT-263 or ABT-737 in hypoxic tumor models $[15,16]$, and as pointed out by Harrison and coworkers [16], whether Mcl-1 is up- or down-regulated may be cell type- and oxygen concentration-dependent. To our knowledge, little information is currently available on 
effects of AZD8055 or other mTOR inhibitors under hypoxic conditions, with the exception of BEZ235 which has been shown to sensitize hypoxic breast and prostate cancer cells to radiation $[26,27]$.

Invariably, CRC comprises heterogeneous tumors with predominant hypoxic components [4], which is important to take into consideration with established as well as novel therapies. The present study showed that comboRx significantly suppressed viability of hypoxic CRC cells, but in six of 14 cell lines there was no additional inhibitory effect of ABT-737 to that of single-agent AZD8055. A similar finding was obtained with the combination of ABT-737 and BEZ235 (potentiation was not seen in five of 12 cell lines) and when AZD8055 in a lower concentration of $0.10 \mu \mathrm{M}$ was given together with ABT-737 (four of seven hypoxic cell lines were resistant to an additional effect of the anti-apoptotic inhibitor). In the five cell lines where mechanistic investigations were undertaken (the KRAS-mutant HCT-116, HCT-15, and LoVo models and the BRAF/PIK3CA-mutant RKO and wild-type Colo320DM cells), combo-Rx under hypoxic conditions caused dual phenotypic responses in terms of concurrent apoptotic and pro-survival effects in the HCT-116 and HCT-15 cell lines, as demonstrated through the specific examination of the anti-apoptotic Mcl-1 protein, microscopy of cell cultures, and both targeted and comprehensive analysis of kinase signaling. Importantly, both of these cell lines also harbor PIK3CA mutation. These findings suggest that $\mathrm{CRC}$ cancers with co-occurring KRAS and PIK3CA mutations, which is not a frequent entity [5], may be particularly susceptible to parallel apoptotic and pro-survival effects with this combination treatment.

The MAPK and PI3K-AKT signaling pathways merge at the mTOR complex, which promotes cell survival through phosphorylation of S6 and the resulting increase in Mcl-1 protein translation [28]. Intriguingly, in hypoxic HCT-116 and HCT-15 cells, combo-Rx strongly increased expression of Mcl-1 and p-S6, which under normoxia and in agreement with previously reported data [9] showed the opposite response following the addition of AZD8055 to ABT-737. The Tyrosine Kinase PamChip Array approach enabled the investigation of more general kinase activity responses. Using this technology, ABT-737 was shown to repress kinase activities known to be important for CRC survival in hypoxic HCT-116 cells. Under normoxic conditions, ABT-737 treatment has been shown to sensitize CRC and rhabdomyosarcoma cell lines for AZD8055directed apoptosis $[9,29]$. It is therefore notable that in our experimental setups, a number of array substrates associated with MAPK/PI3K-conducted signaling (RASA1, RAF1, PIK3R1, and PDPK1) were phosphorylated by lysates from hypoxic HCT-116 cells given combo-Rx. Moreover, array substrates reflecting proteins that are fundamental in the angiogenic response to hypoxia, particularly PDGFRB, were also highly phosphorylated. In angiogenesis, PDGFR is required for the formation of a functional pericyte coverage of regenerating endothelium within the tumor stroma [30]. Importantly, the KRAS-mutant HCC2998 cell line, which was sensitive to combo-Rx for viability but devoid of PIK3CA mutation, and the resistant wild-type Colo320DM model, showed repressed or unchanged global kinase activities to the experimental perturbations. Again, the findings indicate a particular susceptibility of KRAS/PIK3CA-mutant CRC entities to unfavorable responses to the combined inhibition of antiapoptotic proteins and mTOR signaling. Of final note, in the context of interpreting the ex vivo kinase substrate data, some important considerations should be kept in mind [4]. One is that hypoxia elicits a multitude of adaptive signaling responses, which as such are challenging to portray, and another is that phosphorylation of each individual substrate on the kinase target array reflects the net result of an extensive network of multiple kinase activities.

\section{Conclusions}

To conclude, under hypoxia, which is an important feature of CRC tumors and a main mechanism of therapy resistance, the apparently rational approach of combining ABT-737 for inhibition of anti-apoptotic proteins with the mTOR inhibitor AZD8055 [9] in CRC cell lines with cooccurring KRAS and PIK3CA mutations revealed complex responses of pro-survival effects elicited in parallel with apoptosis. Recognizing the obvious limitation that CRC cell lines do not fully reflect the heterogeneity of this disease with regard to mutation profiles or the dynamic nature of tumor oxygenation status, more extensive preclinical studies are required for further elucidation of responses to this kind of combination treatment. Still, from a clinical perspective, and particularly with tumor hypoxia posting a demand for more tailored therapies [31], caution should be practiced on implementation of combinatory strategies.

\section{Additional files}

Additional file 1: Fig. S1. Cell viability of hypoxic colorectal carcinoma (CRC) cell lines. a. Four CRC cell lines were treated for $24 \mathrm{~h}$ with the indicated concentrations of ABT-737 (inhibitor of anti-apoptotic BCl-2 family proteins) or AZD8055 (mTOR inhibitor) under normoxic or hypoxic culture conditions. Cell viability (measured by the MTS assay) value for each condition relative to the corresponding control cell (vehicle-treated) value is shown as mean \pm SD from at least three independent experiments, each plated at least in triplicate. Statistically significant changes are indicated (asterisk, $p<0.05$ cross, $p<0.01$; circle, $p<0.001$ ). b. Cultures of the CRC cell line were left in the hypoxic chamber at $0.2 \% \mathrm{O}_{2}$ and harvested after the indicated time periods. Expression of the hypoxia-inducible factor type $1 a$ (HIF-1a) and its target gene carbonic anhydrase IX (CAIX) was examined by Western blot analysis, with a-tubulin expression as loading control, and with $24 \mathrm{~h}$ of normoxia and $4 \mathrm{~h}$ of $100 \mu \mathrm{M} \mathrm{CoCl}_{2}$ exposure in normoxia to generate 
negative and positive biological controls for HIF-1a expression, respectively. (DOCX $369 \mathrm{~kb}$ )

Additional file 2: Table S1a. Cell viability of normoxic colorectal carcinoma cell lines. From at least three independent experiments, each at least with triplicate setups, mean \pm SD cell viability values were calculated in percentage of values from the corresponding controls (vehicle-treated cells). Treatments (72-h incubations): ABT-737 (inhibitor of anti-apoptotic Bcl-2 family proteins; $10 \mu \mathrm{M}$ ), AZD8055 (mTOR inhibitor; $0.10 \mu \mathrm{M}$ ), both compounds in combination. Difference in values from cells given AZD8055 or the combination treatment was compared by two-tailed Student's $t$-test. Mutation status is indicated for each cell line. Table S1b. Cell viability of hypoxic colorectal carcinoma cell lines. From at least three independent experiments, each at least with triplicate setups, mean \pm SD cell viability values were calculated in percentage of values from the corresponding controls (vehicle-treated cells). Treatments (72-h incubations): ABT-737 (inhibitor of anti-apoptotic Bcl-2 family proteins; $10 \mu \mathrm{M}$ ), AZD8055 (mTOR inhibitor; $0.10 \mu \mathrm{M}$ ), both compounds in combination. Difference in values from cells given AZD8055 or the combination treatment was compared by two-tailed Student's $t$-test. Mutation status is indicated for each cell line. Table S2. Cell viability of normoxic colorectal carcinoma cell lines. From between three and seven independent experiments, each at least with triplicate setups, mean \pm SD cell viability values were calculated in percentage of values from the corresponding controls (vehicle-treated cells). Treatments (24-h incubations): ABT-737 (inhibitor of anti-apoptotic BCl-2 family proteins; $10 \mu \mathrm{M}$ ), AZD8055 (mTOR inhibitor; $10 \mu \mathrm{M}$ ), both compounds in combination (combo-Rx). Difference in values from cells given AZD8055 or combo-Rx was compared by two-tailed Student's $t$-test. Mutation status is indicated for each cell line. Table S3a. Cell viability of normoxic colorectal carcinoma cell lines. From at least three independent experiments, each at least with triplicate setups, mean \pm SD cell viability values were calculated in percentage of values from the corresponding controls (vehicle-treated cells). Treatments (24-h incubations): ABT-737 (inhibitor of anti-apoptotic BCl-2 family proteins; $10 \mu \mathrm{M}$ ), BEZ235 (PI3K mTOR inhibitor; $0.10 \mu \mathrm{M}$ ), both compounds in combination. Difference in values from cells given BEZ235 or the combination treatment was compared by two-tailed Student's $t$-test. Mutation status is indicated for each cell line. Table S3b. Cell viability of hypoxic colorectal carcinoma cell lines. From at least three independent experiments, each at least with triplicate setups, mean \pm SD cell viability values were calculated in percentage of values from the corresponding controls (vehicle-treated cells). Treatments (24-h incubations): ABT-737 (inhibitor of anti-apoptotic Bcl-2 family proteins; $10 \mu \mathrm{M}$ ), BEZ235 (PI3K/mTOR inhibitor; $0.10 \mu \mathrm{M}$ ), both compounds in combination. Difference in values from cells given BEZ235 or the combination treatment was compared by two-tailed Student's t-test. Mutation status is indicated for each cell line. (DOCX $36 \mathrm{~kb})$

Additional file 3: Fig. S2. Microscopy of cellular phenotypes under hypoxia. The three colorectal carcinoma cell lines were given combo-Rx (combination of 10 MM ABT-737, an inhibitor of anti-apoptotic Bcl-2 family proteins, and $10 \mu \mathrm{M}$ AZD8055, an mTOR inhibitor) for $72 \mathrm{~h}$ under hypoxia and inspected by phase-contrast microscopy at 24-h intervals. Control cells received vehicle only. Floating pycnotic cells in medium are indicated by arrows. Scale bars: $50 \mu \mathrm{m}$. (DOCX $1104 \mathrm{~kb}$ )

Additional file 4: Table S4. Tyrosine Kinase PamChip ${ }^{\oplus}$ Array substrates_-phosphorylation levels. The color map visualizes normalized $\log _{2}$-transformed signal intensities from kinase substrate arrays incubated with lysates from the three colorectal carcinoma cell lines treated for $24 \mathrm{~h}$ with ABT-737 (inhibitor of anti-apoptotic Bcl-2 family proteins; $10 \mu \mathrm{M}$ ), AZD8055 (mTOR inhibitor; $10 \mu \mathrm{M}$ ), or combo-Rx (10 $\mu \mathrm{M}$ of both compounds in combination). Red corresponds to higher and blue to lower substrate phosphorylation levels relative to levels from the corresponding control cells. ${ }^{a}$ Retrieved from UniProtKB/SwissProt (http://www.uniprot.org/).

${ }^{b}$ Position(s) of the tyrosine phosphorylation site(s) within the protein. c Retrieved from PathCard (http://pathcards.genecards.org/). Super-pathway definitions were 'PI3K-AKT signaling pathway' and 'MAPK signaling pathway'. (DOC $536 \mathrm{~kb})$

\section{Abbreviations}

CAIX: Carbonic anhydrase IX; CRC: Colorectal carcinoma; ERK1/2: Mitogenactivated protein kinase 3/1; HIF-1a: Hypoxia-inducible factor type 1a; MAPK Mitogen-activated protein kinases; mTOR: Mechanistic target of rapamycin;
PI3K-AKT: Phosphatidylinositol 3-kinase-protein kinase B; S6: Ribosomal protein 56 kinase beta-2; sh: Short hairpin

\section{Acknowledgments}

Not applicable.

\section{Funding}

This study was supported by the Norwegian Cancer Society (Grants 2180105 and 6803027), South-Eastern Norway Regional Health Authority (Grants 2012002, 2014010, and 2014012-ACREDIT), Research Council of Norway (Grant 218325-MetAction), and Akershus University Hospital (Grants 2014028 and 2015003).

\section{Availability of data and materials}

The primary array data are available in the ArrayExpress data repository (http://www.ebi.ac.uk/arrayexpress/experiments/E-MTAB-3870/) by accession number E-MTAB-3870.

\section{Authors' contributions}

KR conceived the study and carried out the molecular analyses. KRR, TB, and JS contributed to the development and validation of technical procedures. KRR and LS undertook the statistical analyses. KR and AHR drafted the manuscript. All authors read and approved the final manuscript.

\section{Authors' information}

KR is Postdoctoral Research Fellow. KRR is Senior Scientist. LS and JS are Research Engineers. TB is Postgraduate Research Fellow. AHR is Clinician-Scientist and Principal Investigator.

\section{Competing interests}

The authors declare that they have no competing interests.

\section{Consent for publication}

Not applicable.

Ethics approval and consent to participate

Not applicable.

\section{Author details}

'Department of Oncology, Akershus University Hospital, 1478 Lørenskog, Norway. ${ }^{2}$ Institute of Clinical Molecular Biology, Akershus University Hospital, 1478 Lørenskog, Norway. ${ }^{3}$ Institute of Clinical Medicine, University of Oslo, 0318 Oslo, Norway.

Received: 9 November 2015 Accepted: 22 July 2016

Published online: 26 July 2016

\section{References}

1. Bristow RG, Hill RP. Hypoxia and metabolism. Hypoxia, DNA repair and genetic instability. Nat Rev Cancer. 2008:8:180-92.

2. Luoto KR, Kumareswaran R, Bristow RG. Tumor hypoxia as a driving force in genetic instability. Genome Integr. 2013;4:5.

3. Wouters BG, Koritzinsky M. Hypoxia signalling through mTOR and the unfolded protein response in cancer. Nat Rev Cancer. 2008;8:851-64.

4. Ree AH, Flatmark K, Saelen MG, Folkvord S, Dueland S, Geisler J, et al. Tumor phosphatidylinositol 3-kinase signaling in therapy resistance and metastatic dissemination of rectal cancer: opportunities for signaling-adapted therapies. Crit Rev Oncol Hematol. 2015;95:114-24.

5. Cancer Genome Atlas Network. Comprehensive molecular characterization of human colon and rectal cancer. Nature. 2012;487:330-7.

6. Laplante M, Sabatini DM. mTOR signaling at a glance. J Cell Sci. 2009;122:3589-94.

7. Francipane MG, Lagasse E. mTOR pathway in colorectal cancer: an update. Oncotarget. 2014:5:49-66.

8. Wang XW, Zhang YJ. Targeting mTOR network in colorectal cancer therapy. World J Gastroenterol. 2014;20:4178-88,

9. Faber AC, Coffee EM, Costa C, Dastur A, Ebi H, Hata AN, et al. mTOR inhibition specifically sensitizes colorectal cancers with KRAS or BRAF mutations to BCL-2/ BCL-XL inhibition by suppressing MCL-1. Cancer Discov. 2014;4:42-52.

10. Oltersdorf T, Elmore SW, Shoemaker AR, Armstrong RC, Augeri DJ, Belli BA, et al. An inhibitor of $\mathrm{BCl}-2$ family proteins induces regression of solid tumours. Nature. 2005;435:677-81. 
11. Billard C. BH3 mimetics: status of the field and new developments. Mol Cancer Ther. 2013;12:1691-700.

12. Konopleva M, Contractor R, Tsao T, Samudio I, Ruvolo PP, Kitada S, et al. Mechanisms of apoptosis sensitivity and resistance to the $\mathrm{BH} 3$ mimetic ABT-737 in acute myeloid leukemia. Cancer Cell. 2006;10:375-88.

13. van Delft MF, Wei AH, Mason KD, Vandenberg CJ, Chen L, Czabotar PE, et al. The $\mathrm{BH} 3$ mimetic ABT-737 targets selective $\mathrm{BCl}-2$ proteins and efficiently induces apoptosis via Bak/Bax if Mcl-1 is neutralized. Cancer Cell. 2006;10:389-99.

14. Risberg K, Fodstad O, Andersson Y. Synergistic anticancer effects of the 9.2. 27PE immunotoxin and ABT-737 in melanoma. PLoS One. 2011;6, e24012.

15. Klymenko T, Brandenburg M, Morrow C, Dive C, Makin G. The novel BCl-2 inhibitor ABT-737 is more effective in hypoxia and is able to reverse hypoxia-induced drug resistance in neuroblastoma cells. Mol Cancer Ther. 2011;10:2373-83.

16. Harrison LR, Micha D, Brandenburg M, Simpson KL, Morrow CJ, Denneny O, et al. Hypoxic human cancer cells are sensitized to $\mathrm{BH}-3$ mimetic-induced apoptosis via downregulation of the BCl-2 protein Mcl-1. J Clin Invest. 2011; 121:1075-87.

17. Huelsemann MF, Patz M, Beckmann L, Brinkmann K, Otto T, Fandrey J, et al. Hypoxia-induced p38 MAPK activation reduces Mcl-1 expression and facilitates sensitivity towards BH3 mimetics in chronic lymphocytic leukemia. Leukemia. 2015:29:981-4.

18. Ahmed D, Eide PW, Eilertsen IA, Danielsen SA, Eknaes M, Hektoen M, et al. Epigenetic and genetic features of 24 colon cancer cell lines. Oncogenesis. 2013;2, e71.

19. Edkins S, O'Meara S, Parker A, Stevens C, Reis M, Jones S, et al. Recurrent KRAS codon 146 mutations in human colorectal cancer. Cancer Biol Ther. 2006:5:928-32.

20. Ikediobi ON, Davies H, Bignell G, Edkins S, Stevens C, O'Meara S, et al. Mutation analysis of 24 known cancer genes in the NCl-60 cell line set. Mol Cancer Ther. 2006;5:2606-12.

21. Tahiri A, Roe K, Ree AH, de Wijn R, Risberg K, Busch C, et al. Differential inhibition of ex-vivo tumor kinase activity by vemurafenib in BRAF(V600E) and BRAF wild-type metastatic malignant melanoma. PLoS One. 2013;8, e72692.

22. Brachmann SM, Hofmann I, Schnell C, Fritsch C, Wee S, Lane H, et al. Specific apoptosis induction by the dual PI3K/mTor inhibitor NVP-BEZ235 in HER2 amplified and PIK3CA mutant breast cancer cells. Proc Natl Acad Sci U S A. 2009;106:22299-304.

23. Manara MC, Nicoletti G, Zambelli D, Ventura S, Guerzoni C, Landuzzi L, et al. NVP-BEZ235 as a new therapeutic option for sarcomas. Clin Cancer Res, 2010;16:530-40.

24. Kroemer G, Galluzzi L, Vandenabeele P, Abrams J, Alnemri ES, Baehrecke EH, et al. Classification of cell death: recommendations of the nomenclature committee on cell death 2009. Cell Death Differ. 2009:16:3-11.

25. Huang Z, Wu Y, Zhou X, Qian J, Zhu W, Shu Y, et al. Clinical efficacy of mTOR inhibitors in solid tumors: a systematic review. Future Oncol. 2015;11:1687-99.

26. Kuger S, Corek E, Polat B, Kammerer U, Flentje M, Djuzenova CS. Novel PI3K and mTOR inhibitor NVP-BEZ235 radiosensitizes breast cancer cell lines under normoxic and hypoxic conditions. Breast Cancer (Auckl). 2014;8:39-49.

27. Potiron VA, Abderrahmani R, Giang E, Chiavassa S, Di Tomaso E, Maira SM, et al. Radiosensitization of prostate cancer cells by the dual PI3K/mTOR inhibitor BEZ235 under normoxic and hypoxic conditions. Radiother Oncol. 2013;106:138-46.

28. Mills JR, Hippo Y, Robert F, Chen SM, Malina A, Lin CJ, et al. mTORC1 promotes survival through translational control of Mcl-1. Proc Natl Acad Sci U S A. 2008;105:10853-8.

29. Preuss E, Hugle M, Reimann R, Schlecht M, Fulda S. Pan-mammalian target of rapamycin (mTOR) inhibitor AZD8055 primes rhabdomyosarcoma cells for ABT-737-induced apoptosis by down-regulating Mcl-1 protein. J Biol Chem. 2013;288:35287-96.

30. Casazza A, Di Conza G, Wenes M, Finisguerra V, Deschoemaeker S, Mazzone M. Tumor stroma: a complexity dictated by the hypoxic tumor microenvironment. Oncogene. 2014;33:1743-54.

31. Vaupel P, Mayer A. The clinical importance of assessing tumor hypoxia: relationship of tumor hypoxia to prognosis and therapeutic opportunities. Antioxid Redox Signal. 2015;22:878-80.

\section{Submit your next manuscript to BioMed Central and we will help you at every step:}

- We accept pre-submission inquiries

- Our selector tool helps you to find the most relevant journal

- We provide round the clock customer support

- Convenient online submission

- Thorough peer review

- Inclusion in PubMed and all major indexing services

- Maximum visibility for your research

Submit your manuscript at www.biomedcentral.com/submit
Biomed Central 